\title{
Perspectiva de los egresados como facilitadores en simulación dentro del Centro de Enseñanza y Certificación de Aptitudes Médicas
}

\author{
Perspective of the graduates as facilitators in simulation within \\ the Center for Teaching and Certification of Medical Skills \\ Hugo Erick Olvera-Cortés,* Sara Morales-López,* \\ Samantha López-González,* Rosa María Leal-Farfán*
}

Palabras clave:

Simulación,

entrenamiento,

facilitador, encuesta,

instructor.

Keywords:

Simulation, training, facilitator, survey, instructor.

\footnotetext{
* Académico del Centro de Enseñanza y Certificación de Aptitudes Médicas, Departamento de Integración de Ciencias Médicas, Facultad de Medicina de la Universidad Nacional Autónoma de México
}

\section{RESUMEN}

Introducción: Para lograr los objetivos de la simulación clínica es necesario contar con facilitadores capacitados. En el caso de la Facultad de Medicina de la Universidad Nacional Autónoma de México, los médicos pasantes de servicio social fungen como facilitadores para los más de cinco mil estudiantes que visitan el centro de simulación mensualmente. Objetivo: Conocer la perspectiva de los médicos pasantes de servicio social acerca de su capacitación para poder desempeñarse como facilitadores de las actividades de aprendizaje con simulación. Material y métodos: Se recabaron las opiniones de los médicos pasantes a través de una encuesta de autopercepción acerca de los diversos temas que incluye el programa de formación del facilitador en simulación del centro, posterior a una capacitación de aproximadamente 260 horas. Resultados y conclusiones: De acuerdo con la percepción de los médicos pasantes, la formación del facilitador es de suma relevancia y completa; sin embargo, se hicieron conscientes de los retos que enfrentan y de la necesidad de que dicha capacitación sea continua. El $76 \%$ se encuentra totalmente de acuerdo en que mejoró sus habilidades no técnicas y $69 \%$ en que mejoró sus habilidades técnicas; $57 \%$ considera que su desempeño fue excelente; 35.8 y 53.8\%, respectivamente, estuvieron totalmente de acuerdo y de acuerdo en volver a realizar su servicio social en el centro de simulación.

\section{ABSTRACT}

Introduction: To achieve the objectives of clinical simulation it is necessary to have trained facilitators. In the case of the Faculty of Medicine of the National Autonomous University of Mexico, the medical interns of social service act as facilitators for the more than five thousand students who visit the simulation center monthly. Objective: To know the perspective of the Interns of Social Service about their training in order to be able to act as facilitators of simulation learning activities. Material and methods: The opinions of the medical interns were gathered through a self-perception survey on the various topics that include the training program of the facilitator in simulation of the center, following an approximate 260 hours of training. Results and conclusions: According to the perception of the trainee doctors, the training of the facilitator is extremely relevant and it has been complete; however, they became aware of the challenges they face and of the need for such training to be continuous. $76 \%$ totally agree that they improved their non-technical skills and $69 \%$ that they improved their technical skills, 57\% consider that their performance was excellent, 35.8 and $53.8 \%$, respectively, totally agreed and agreed to return to perform their social service in the simulation center.

\section{INTRODUCCIÓN}

T a simulación clínica consiste en situar a un $ـ$ estudiante en un contexto que imite algún aspecto de la realidad clínica, tal como lo menciona David Gaba: "Es una técnica, para sustituir o ampliar las experiencias reales, a través de experiencias guiadas, que evocan o replican aspectos sustanciales del mundo real, de una forma totalmente interactiva"; ${ }^{1}$ en medicina, ha sido utilizada para mejorar la capacitación de recursos humanos e impactar directamente en la seguridad del paciente lo que ha disminuido los errores clínicos. ${ }^{2}$ La simulación crea un ambiente ideal para la educación debido a que las actividades pueden diseñarse para que sean predecibles, consistentes, estandarizadas, seguras y reproducibles para llevar a cabo el 
aprendizaje experiencial y favorecer el desarrollo de las competencias. ${ }^{3-5}$

En el contexto español, el rol docente en simulación clínica se conoce como "instructores", "monitores" o "facilitadores"; sin embargo, para fines del presente escrito se tomará como "facilitadores" a médicos que recién terminaron su ciclo académico formal de cinco años y que tendrán que cubrir un año de servicio social en la modalidad de programa universitario, lo cual es un requisito para titulación de la licenciatura.

Los facilitadores tienen la función de guiar la actividad de aprendizaje en simulación para optimizar las oportunidades del participante de desarrollar experiencias lo más cercanas a la realidad a fin de conseguir los objetivos propuestos; asimismo, el facilitador se debe preocupar por poner al estudiante en contacto con las situaciones de aprendizaje para que construya su propia enseñanza con base en las experiencias previas, la búsqueda de conocimientos y la capacidad de reflexión e integración de los nuevos saberes. ${ }^{6-8}$

El facilitador de este proceso de aprendizaje debe tener características importantes, tales como gestionar y evaluar adecuadamente el trabajo grupal e individual en el seno del mismo, estimular la búsqueda activa de información relativa al escenario clínico que han de trabajar, contextualizar los distintos roles que los estudiantes tendrán a la hora de afrontar el escenario clínico, motivar al estudiante a adoptar un papel activo en detrimento del tradicional rol pasivo de otras estrategias, estimular la reflexión "en la acción" y "sobre la acción" por parte de los participantes y observantes, incitar a la participación colectiva del grupo en la observación de lo realizado por los compañeros, entre otras. ${ }^{6,9}$ Para lograr obtener estas características, los facilitadores empezaron a introducirse en la simulación clínica mediante el ensayo de casos simulados en la propia institución, visitando otros centros de trabajo, realizando una rotación electiva en educación médica (que incluye la formación en simulación clínica), participando en talleres, jornadas, cursos o congresos, realizando estancias (pasantías) y obteniendo diversos títulos en simulación. ${ }^{10,11}$

Entre estas modalidades de capacitación existe la estancia académica que es el equiva- lente del anglicismo fellowship, que se utiliza habitualmente en España; no obstante, es más común el término pasantía para referirse a él en Latinoamérica. Ésta consiste en una posición laboral, con fechas fijas de inicio y final, en la cual se ofrece la oportunidad de formación profesional al trabajar con otros profesionales y mentores que brindan tutoría sobre un área o proyecto adaptados a las necesidades del participante. La misión de una pasantía en simulación en salud es facilitar al participante la adquisición de conocimientos, habilidades, actitudes y comportamientos necesarios para concebir, diseñar, implementar, gestionar, evaluar y desarrollar programas que utilicen la simulación como herramienta docente. ${ }^{10,11}$

Dentro del marco de la capacitación, es de suma importancia conocer la percepción que tiene el participante acerca de la capacitación en simulación, con el fin de mejorar las características del programa, el cual es el objetivo del presente trabajo.

\section{MATERIAL Y MÉTODOS}

El presente trabajo desarrolla un estudio cuasiexperimental, realizado en el Centro de Enseñanza y Certificación de Aptitudes Médicas (CECAM), centro de simulación de pregrado de la Facultad de Medicina de la Universidad Nacional Autónoma de México.

\section{Muestra}

El tamaño de la muestra fue el total de la población: 27 médicos pasantes de servicio social (MPSS), los cuales pertenecen al grupo de edad entre los 24 y 26 años. Los MPSS fueron seleccionados para realizar su servicio social en el periodo de febrero de 2018 a enero de 2019 en el centro de simulación, cumpliendo los criterios establecidos en la normatividad universitaria, además de cumplir con los requisitos establecidos por el centro.

\section{Procedimiento}

Los MPSS tienen la función de facilitadores de actividades de aprendizaje con simulación, las cuales son más de 60 diferentes, con distintos niveles de dificultad para los estudiantes desde 
el primer año hasta el quinto año de la carrera de médico cirujano.

\section{Algunos de los roles que deben cumplir son:}

1. Facilitador en aprendizaje con entrenadores de tareas: donde los MPSS realizan el modelado de procedimientos como intubación, colocación de sonda vesical, entre otras, y posteriormente ayuda y guía al participante a dominar la habilidad.

2. Paciente estandarizado: durante algunas actividades con simulación los MPSS simulan ser pacientes.

3. Confederado (personal de enfermería, laboratorio, etcétera): durante escenarios de alta fidelidad donde es necesario tener apoyo de personal de enfermería y personal de laboratorio médico interconsultante, los MPSS realizan esta actividad para guiar a los participantes en el logro de los objetivos.

4. Evaluador en actividades y examen clínico objetivo estructurado (ECOE): durante las

Tabla 1: Sesiones de capacitación y su duración.

\begin{tabular}{|c|c|c|}
\hline Temática & Sesiones & $\begin{array}{c}\text { Duración } \\
\text { total } \\
\text { (horas) }\end{array}$ \\
\hline Capacitaciones teóricas & 30 & 30 \\
\hline $\begin{array}{l}\text { Capacitación en simulación } \\
\text { - Simulación definiciones } \\
\text { - Tipo de simuladores } \\
\text { - Etapas de la simulación } \\
\text { - Facilitador en simulación } \\
\text { - Debriefing } \\
\text { - Ruidos y señales } \\
\text { - Aprendizaje con entrenadores de tareas }\end{array}$ & 80 & 80 \\
\hline Capacitación en logísticas de las actividades & 50 & 50 \\
\hline Paciente estandarizado y manejo de emociones & 10 & 20 \\
\hline Manejo del estrés, resiliencia y trabajo en equipo & 10 & 20 \\
\hline Investigación en simulación & $\begin{array}{c}1 \text { presencial } \\
\text { y curso en } \\
\text { línea }\end{array}$ & 20 \\
\hline Uso de simuladores & 20 & 40 \\
\hline Autoprotección & 3 & 6 \\
\hline Evaluador & 15 & 20 \\
\hline
\end{tabular}

actividades de simulación y ECOE, los MPSS realizan actividades de evaluadores con el empleo de lista de cotejo, de apreciación y rúbricas.

5. Debriefer/Co-debriefer: es una de las actividades más importantes dentro de la simulación; posterior al escenario, los MPSS guían a los participantes dentro del proceso del debriefing.

6. Realizar un proyecto de investigación: durante la estancia en el centro de simulación los MPSS deben diseñar y poner en práctica un proyecto de investigación educativa.

Para poder cumplir con las funciones antes mencionadas, durante el año de su servicio social reciben una capacitación exhaustiva con el fin de que las actividades sean impartidas con una alta calidad, dicha capacitación se centra en diferentes áreas, las cuales se muestran en la Tabla 1.

Los MPSS reciben una capacitación inicial y comienzan sus actividades dentro del centro de simulación. A lo largo del año de su servicio social reciben capacitación continua en simulación clínica con los apartados ya mencionados. Todas las capacitaciones se centran $20 \%$ en teoría y $80 \%$ en actividades prácticas que les permiten desarrollar las habilidades necesarias para ser facilitadores en simulación de los diferentes escenarios.

Se comienza con un curso de inducción intensivo de 10 días de duración, donde se capacitan en diferentes habilidades. Posteriormente, previo a cada actividad con simulación diferente, los MPSS reciben una capacitación teórica impartida por un experto en el tema de la actividad con simulación; esto ayuda a que los médicos pasantes complementen la información adquirida en su formación, además de resolver dudas. Después de la capacitación teórica, los MPSS viven la actividad con el rol de estudiantes; ésta es impartida por personal del centro con mayor experiencia en este campo. Una vez concluida la etapa anterior, se realiza una capacitación sobre la logística con la que se debe de impartir la actividad: tiempos, estructura, materiales necesarios, roles, entre otros; finalmente, se realiza un ensayo de la actividad donde se provee un feedback de desempeño para poder mejorar el estar frente a un grupo de estudiantes. 
Parte importante de las funciones del MPSS es ser paciente estandarizado en diferentes escenarios, por lo que debe comprender el concepto y tener todas las características que sean necesarias para dicha actividad; esta capacitación es impartida por un profesor de teatro que realiza diversas actividades con ellos, lo cual permite desarrollar su inteligencia emocional, el lenguaje no verbal al interpretar un paciente y caracterizar la misma emoción que debería tener un paciente en ese momento. Debido a la cantidad de actividades que tienen los MPSS, el contacto con diferentes actitudes de los estudiantes y la importancia de tener actividades con simulación de calidad, los médicos pasantes reciben capacitación en manejo del estrés, resiliencia y trabajo en equipo por parte del departamento de psicología, lo cual ayuda a mejorar su capacidad para poder responder mejor a las demandas y eventualidades del centro.

Como parte de su servicio social, el médico pasante debe realizar un proyecto de investigación orientado a la simulación clínica. Esta capacitación queda a cargo de la coordinación de investigación del departamento; ésta se divide en una sesión inicial presencial de las bases de la investigación, posteriormente, se realiza un curso en línea asincrónico con el fin de ayudarlos a desarrollar su proyecto paso a paso, y después, toman asesorías personalizadas con personal capacitado en investigación en simulación.

En el Centro de Simulación se cuenta con más de 300 simuladores con más de 40 tipos (entre ellos de baja, mediana y alta fidelidad), así que previo a impartir las actividades de aprendizaje con simulación, los MPSS se capacitan en el uso de cada uno de estos simuladores.

Durante sus actividades en el centro de simulación, es necesario estar preparado para protegerse y proteger a los demás en la medida de lo posible, por lo cual reciben capacitaciones de medidas de protección civil, evacuación, medidas en caso de un sismo, detección y control de conatos de incendio, entre otros.

Una más de sus actividades es ser evaluadores, para ello reciben una capacitación teórica de la evaluación, tipos y funciones del evaluador, después realizan ejercicios de simulación con estudiantes difíciles y situaciones difíciles en la evaluación, para posteriormente realizar capacitaciones continuas y simulaciones de evaluación con feedback de un experto.

Al finalizar el año de pasantía, el tiempo total de capacitación para los médicos pasantes fue de 260 horas, esto para poder impartir un aproximado de 500 actividades con simulación, primordialmente en el centro de simulación; además de participar como facilitador en curso de reanimación cardiopulmonar (RCP) básico para comunidad, programa ayudando a respirar al bebé, primeros auxilios, electrocardiografía, entre otros.

Se solicitó a los médicos pasantes que contestaran el cuestionario de manera voluntaria y anónima, a través de un formulario online.; El cuestionario contó con 30 ítems, agrupados en ocho dimensiones teóricas, basadas en la experiencia de los autores sobre las actividades: teóricas, en evaluación, como paciente estandarizado, como facilitador, como debriefer, actividades extras, investigación y satisfacción de desempeño.

Una vez concluida la obtención de datos, se utilizó el programa IBM SPSS Statistics para realizar el análisis estadístico, con el cual se obtuvo un análisis factorial para agrupación de dimensiones, análisis de frecuencias y porcentajes.

\section{RESULTADOS}

Se obtuvo una tasa de respuesta del $100 \%$ de la totalidad de la muestra, ya que todos los médicos pasantes que realizaron su servicio social en el centro de simulación contestaron el cuestionario.

En el análisis por frecuencias en la dimensión de capacitaciones teóricas, capacitación como evaluador, capacitación como paciente estandarizado, como facilitador, como debriefer, actividades extras, investigación y satisfacción de desempeño, se encuentran expresados en la Tabla 2.

En cuanto a las capacitaciones teóricas, 7.9 y $61.5 \%$ estuvieron totalmente de acuerdo y de acuerdo, respectivamente. Respecto a si consideraban que la duración de las sesiones fue adecuada, 19.2\% estuvo totalmente de acuerdo y $57.7 \%$ estuvo de acuerdo, los cuales son índices altos; sin embargo, existe un porcentaje representativo que considera que el número y la duración no son suficiente. 
Respecto a su capacitación como facilitador, $38.5 \%$ estuvo totalmente de acuerdo y $42.3 \%$ estuvo de acuerdo en que fue suficiente en duración. Sobre el número de sesiones, 38.5\% estuvo totalmente de acuerdo y $34.6 \%$ estuvo de acuerdo; a diferencia de la capacitación en debriefing donde $26.9 \%$ estuvo totalmente de acuerdo y $38.5 \%$ estuvo de acuerdo en que la capacitación fue suficiente en número; $30.8 \%$ estuvo totalmente de acuerdo en que la capacitación fue suficiente en tiempo, aunque sólo $7 \%$ se sintió seguro en sus primeros tres

Tabla 2: Porcentaje de respuesta a la encuesta de satisfacción.

\begin{tabular}{|c|c|c|c|c|c|}
\hline & TDA & DA & I & $\mathrm{D}$ & TD \\
\hline Fueron adecuadas en número & 7.9 & 61.5 & 7.7 & 23.1 & 0.0 \\
\hline Fueron adecuadas en duración & 19.2 & 57.7 & 0.0 & 23.1 & 0.0 \\
\hline Tenían relevancia & 42.3 & 38.4 & 0.0 & 15.4 & 3.9 \\
\hline Tuvieron un ponente que desarrolló adecuadamente el tema & 23.1 & 57.7 & 7.7 & 7.7 & 3.9 \\
\hline \multicolumn{6}{|l|}{ - En cuanto a mi capacitación como evaluador considero } \\
\hline Fueron adecuadas en número & 27.0 & 57.7 & 7.7 & 7.7 & 0.0 \\
\hline Fueron adecuadas en duración & 26.7 & 50.0 & 15.4 & 7.7 & 0.0 \\
\hline Tuvo actividades que me ayudaron a desempeñarme adecuadamente con los estudiantes & 26.7 & 46.2 & 26.7 & 0.0 & 0.0 \\
\hline Considero que la evaluación que yo realizaba era similar a la de mis compañeros de estación & 0.0 & 46.2 & 26.9 & 26.9 & 0.0 \\
\hline \multicolumn{6}{|l|}{ - En relación con la capacitación como paciente estandarizado considero } \\
\hline Fueron adecuadas en número & 30.8 & 50.0 & 3.8 & 11.5 & 3.8 \\
\hline Fueron adecuadas en duración & 38.5 & 53.8 & 3.8 & 0.0 & 3.8 \\
\hline Tuvo actividades que me ayudaron a desempeñarme adecuadamente con los estudiantes & 46.2 & 50.0 & 3.8 & 0.0 & 0.0 \\
\hline El profesor de teatro le dio otra perspectiva a ser paciente estandarizado & 61.5 & 30.8 & 7.7 & 0.0 & 0.0 \\
\hline \multicolumn{6}{|l|}{ - En relación a mi capacitación como facilitador en simulación } \\
\hline Me parecieron adecuadas en cuanto a duración & 38.5 & 42.3 & 7.7 & 11.5 & 0.0 \\
\hline Fueron suficientes en número & 38.5 & 34.6 & 11.5 & 15.4 & 0.0 \\
\hline Me sentí seguro al guiar un escenario de simulación de alta fidelidad & 53.8 & 34.6 & 11.5 & 0.0 & 0.0 \\
\hline Me sentí seguro al guiar una actividad de entrenadores de tareas & 53.8 & 46.2 & 0.0 & 0.0 & 0.0 \\
\hline \multicolumn{6}{|l|}{ - En cuanto a capacitación en debriefing } \\
\hline Mi capacitación en debriefing fue suficiente en número & 26.9 & 38.5 & 3.8 & 26.9 & 3.8 \\
\hline Tuvo una duración adecuada & 30.8 & 38.5 & 11.5 & 19.2 & 0.0 \\
\hline En mis primeros tres debriefing me sentí seguro & 7.7 & 34.6 & 15.4 & 42.3 & 0.0 \\
\hline Actualmente me siento seguro para guiar un debriefing & 69.2 & 30.8 & 0.0 & 0.0 & 0.0 \\
\hline \multicolumn{6}{|l|}{ - En relación con las actividades extras } \\
\hline Considero necesario la inclusión de actividades de protección civil & 73.1 & 19.2 & 7.7 & 0.0 & 0.0 \\
\hline Considero necesario la inclusión de actividades sobre manejo de incendios & 69.2 & 30.8 & 0.0 & 0.0 & 0.0 \\
\hline Considero necesario la inclusión de actividades de trabajo en equipo y resiliencia & 65.4 & 26.9 & 7.7 & 0.0 & 0.0 \\
\hline \multicolumn{6}{|l|}{ - Satisfacción de desempeño como facilitador en simulación } \\
\hline Considero que mi desempeño durante el servicio social fue excelente & 57.7 & 38.5 & 3.8 & 0.0 & 0.0 \\
\hline Hacer el servicio social en CECAM me permitió conocerme & 61.5 & 34.6 & 3.8 & 0.0 & 0.0 \\
\hline Hacer el servicio social el CECAM fue una experiencia agradable & 42.3 & 53.8 & 3.8 & 0.0 & 0.0 \\
\hline Volvería a realizar mi servicio social en CECAM & 38.5 & 53.8 & 7.7 & 0.0 & 0.0 \\
\hline Recomendaría esta sede & 38.5 & 53.8 & 7.7 & 0.0 & 0.0 \\
\hline Hacer el servicio social en CECAM fortaleció mis habilidades técnicas & 69.2 & 23.1 & 7.7 & 0.0 & 0.0 \\
\hline Hacer el servicio social en CECAM fortaleció mis habilidades no técnicas & 76.9 & 19.2 & 3.8 & 0.0 & 0.0 \\
\hline Me siento más seguro al hablar en público & 80.8 & 19.2 & 0.0 & 0.0 & 0.0 \\
\hline
\end{tabular}

TDA = Totalmente de acuerdo, DA = De acuerdo, $\mathrm{I}$ = Indiferente, $\mathrm{D}$ = Desacuerdo, $\mathrm{TD}$ = Totalmente en desacuerdo. 
debriefing; después de realizar cerca de 200 debriefing, $69.2 \%$ estaba totalmente de acuerdo en sentirse seguro al guiar un debriefing.

Por otra parte, $57.7 \%$ está totalmente de acuerdo y $30 \%$ está de acuerdo en que su desempeño durante su año de servicio social fue excelente. En el caso de recomendar la sede como lugar de servicio social, $38.5 \%$ estuvo totalmente de acuerdo y 53.8\% respondió estar de acuerdo.

En cuanto al espacio para comentarios se describió lo siguiente para cada una de las dimensiones: para las capacitaciones teóricas, 15 participantes indicaron que era recomendable aumentar el tiempo y 10 mencionaron grabar las ponencias para poder revisarlas posteriormente; en la capacitación como paciente estandarizado, 18 participantes señalaron que es necesaria una evaluación continua. En cuanto a la capacitación como facilitador de las actividades de aprendizaje, 10 estudiantes mencionaron que se incluya el tema de manejo del estudiante difícil capacitación como debriefer, siete participantes comentaron que se aumentara el número y 12 mencionaron que se realizará una evaluación continua y, a partir de esto, realizar un feedback sobre el debriefing. Respecto a su satisfacción de desempeño mencionaron: "Amo el CECAM, es una experiencia única y especial, hacer mi servicio social en esta sede me permitió crecer y desarrollarme tanto profesional como personalmente y en este ámbito de la docencia". "Sin duda el mejor año de mi carrera. Fue positivo en cuanto a formación profesional, como docente y con el mejor equipo de trabajo y en el mejor ambiente". "En definitiva el médico que entró al CECAM y el que se va es muy diferente. Creo que he evolucionado para bien y he aprendido un montón. El CECAM cumplió todas mis expectativas y las sobrepasó. Creo que tuve mucha suerte de poder hacer lo que amo y conocer más cada día".

\section{DISCUSIÓN}

Posterior a que cada MPSS impartiera más de 500 actividades de aprendizaje con simulación y recibiera más de 200 horas de capacitación, de acuerdo con los resultados de la encuesta, aproximadamente $30 \%$ considera que las capacitaciones no son suficientes en número y en duración, esto debido a la gran cantidad de temas que se deben de cubrir durante su estancia en el centro, por lo que no pueden tener capacitaciones exhaustivas en un tema, sino que el programa está desarrollado con el fin de que logren las habilidades en corto tiempo y que puedan impartir las actividades con la calidad necesaria; por ejemplo, en cuanto a la capacitación de debriefing 30\% considera que no es suficiente en un número y tiempo y en los primeros tres debriefings no se sintieron seguros, pero al término de su pasantía 100\% se encontraba de acuerdo y totalmente de acuerdo en que podían guiar un debriefing.

Cabe señalar que no hay publicaciones respecto a la satisfacción de los MPSS sobre su experiencia como facilitador o su capacitación en un centro de simulación, por lo tanto, es necesario realizar una mayor investigación sobre estos temas y las competencias específicas que debería tener el facilitador en simulación y cuál es la forma más efectiva para poder desarrollarlas.

El médico pasante como facilitador en actividades de aprendizaje con simulación tiene grandes ventajas, pero también desventajas, y una de ellas es que después de un periodo prolongado de capacitación continua concluye su servicio social y éste debe dejar el Centro de Simulación después de un año, por lo que es necesario comenzar con la capacitación de los nuevos médicos pasantes, esto a su vez no ha ayudado a mejorar el programa de capacitación año con año.

En la actualidad, no existen publicaciones sobre las evaluaciones en cuanto a la capacitación de MPSS, ni en evaluación de capacitación a largo plazo; sin embargo, existen evaluaciones de cursos cortos, tales como la evaluación del curso de instructores de la Universidad Católica de Murcia, la cual se centra en la satisfacción del curso mediante una encuesta de satisfacción basada en tres dimensiones: planificación de la docencia, desarrollo de la enseñanza y resultado; no obstante, los instrumentos usados no son equiparables. Otro punto relevante es que dicho estudio evalúa las habilidades del participante posterior a la intervención, situación que es considerada para realizar en próximas investigaciones. 


\section{CONCLUSIONES}

En un centro de simulación lo más importante son los recursos humanos, por lo que cada centro debe invertir en la capacitación continua de estas personas. El tipo de capacitación puede ser en diferentes modalidades; sin embargo, éste no es el único factor, el tiempo y las estrategias de capacitación resultan un punto importante, tanto en la efectividad como en la conformidad del participante sobre la capacitación.

De acuerdo con las opiniones de los participantes, las capacitaciones deben ser en un mayor número y tiempo, todo en relación con las actividades que realizará el facilitador en simulación, así ayudará a determinar cuál será el tiempo que debe capacitarse. Otro punto determinante son los estándares que tendrá que cumplir el personal antes de comenzar a tener contacto con los estudiantes.

Entre las limitaciones de la investigación es preciso mencionar que, si bien la metodología permite capacitar a los facilitadores del centro de simulación ya mencionado, encontrando una aceptación por parte de ellos, es necesario considerar que el programa de capacitación deberá ser modificado de acuerdo con cada centro de simulación y las funciones de los facilitadores dentro de éste.

En futuras propuestas de investigación, a partir de los resultados obtenidos, se pretende extender el presente estudio a los diferentes centros de simulación de México que cuente con MPSS, así como realizar una evaluación de sus habilidades posterior a la capacitación y conocer la percepción de los estudiantes con los que tienen contacto.

\section{AGRADECIMIENTOS}

Agradecemos a todos los médicos pasantes que han realizado su servicio social en el centro de simulación.

\section{REFERENCIAS}

1. Gaba DM. The future vision of simulation in health care. Qual Saf Health Care. 2004; 13 Suppl 1: i2-i10.

2. Corvetto M, Bravo MP, Montaña R, Utili F, Escudero E, Boza C, et al. Simulación en educación médica: una sinopsis. Rev Med Chile. 2013; 141: 70-79.

3. Ferrero F. ¿Puede la simulación clínica contribuir al aprendizaje significativo de competencias educativas? Una aproximación constructivista. Rev Fac Med UNAM. 2017; 60 (S1): 49-59.

4. Juguera-Rodríguez L, Díaz-Agea JL, Pérez-Lapuente ML, Leal-Costa C, Rojo-Rojo A, Echevarría-Pérez P. La simulación clínica como herramienta pedagógica: percepción de los alumnos de Grado en Enfermería en la UCAM (Universidad Católica San Antonio de Murcia). Enferm Glob. 2014; 13 (33): 175-190.

5. Rojo A, Díaz JL. El rol del instructor de simulación clínica. En: Muñoz-Carril PC, Raposo-Rivas M, González-Sanmamed M, Martínez-Figueira ME, Zabalza-Cerdeiriña M, Pérez-Abellás A. Un practicum para la formación integral de los estudiantes. Santiago de Compostela: ANDAVIRA; 2013. pp. 1485-1492.

6. Urra-Medina E, Sandoval-Barrientos S, IrribarrenNavarro F. El desafío y futuro de la simulación como estrategia de enseñanza en enfermería. Investigación Educ Médica. 2017; 6 (22): 119-125.

7. Leal-Costa $C$, Juguera-Rodríguez L, Pardo-Ríos $M$, Martín-Robles MR, Díaz-Agea JL. Evaluación del curso de instructores en simulación clínica de la Universidad Católica de Murcia (UCAM). Revista Enfermería Docente. 2015; (103): 8-14.

8. Maestre JM, Szyld D, del Moral I, Ortiz G, Rudolph JW. La formación de expertos clínicos: la práctica reflexiva. Rev Clin Esp. 2014; 214: 216-220.

9. Zabalza MA, Beraza MAZ. Competencias docentes del profesorado universitario: calidad y desarrollo profesional. Madrid: Narcea Ediciones; 2003.

10. Opazo I, Rojo E, Maestre J. Modalidades de formación de instructores en simulación clínica: el papel de una estancia o pasantía, Rev Educ Med. 2017; 18 (1): 22-29.

11. López-Doueil M, Del Moral I, Maestre JM. Elective rotation in simulation-based medical education during anesthesia residency. Rev Esp Anestesiol Reanim. 2016; 63 (3): 181-182.
Correspondencia:

Hugo Erick Olvera-Cortés

Av. Universidad Núm. 3000,

Ciudad Universitaria, 04510,

Alcaldía Coyoacán, Ciudad de México.

E-mail: hugo.eoc1@gmail.com 